\section{A strange discipline. . .}

SIR - The review article "Strange kin" etics" by Shlesinger et al. "explores the remarkable characteristics of complex, nonlinear processes evolving in space and time. The authors anticipate that a kinetic description of nonlinear dynamics will generate insights into a wide range of complex systems that evolve along interesting trajectories representing deterministic chaos. By coincidence it follows a thoughtful view ${ }^{2}$ of the first 40 years of molecular biology. The spectacular advances along the trajectory of this field have been blemished in unexpected ways that escape an easy explanation. May not strange kinetics provide new insights into, or even remedies for, the shortcomings of molecular biology?

The shortcomings of molecular biology referred to may be side-effects of rapid progress: (1) Preoccupation with enumeration of molecular components without quantifying the processes involved. (2) A tendency by molecular biologists not to reflect on the significance of their data, but to focus on the next piece in one of the puzzles. (3) A reward system that encourages competition between colleagues with almost identical aims and skills.

How are nonlinear dynamical systems relevant? The evolution of a complex field such as molecular biology over a 40-year period would be expected to have a strange trajectory. Its behaviour and the new structures it generates would be at least as complex as those generated by the strange kinetics of simpler systems. Its trajectory would remain sensitive to the initial conditions under which it developed, themselves greatly influenced by theoretical considerations and by the technologies of the physical sciences. Only after a series of spectacular discoveries on the structure of DNA, the specificity of base pairing and the intricacies of gene expression did molecular biology mature around what might be called it own formalisms.

The observed specificity of molecular biology sets the field apart from the older disciplines of physiology and medicine, which often depended on a linear analysis of precisely measured flows in and out of a compartment defined by the investigator. In the kidney, for example, balance and clearance studies still serve to quantify its overall operations. Even at the epithelial and cellular levels, studies of transport have remained highly quantitative. When nonequilibrium thermodynamics was introduced to the study of irreversible processes, transport physiologists applied linear forms of analysis based on formalisms for near-equilibrium conditions ${ }^{3,4}$.

These formalisms, however, were too restrictive for molecular biologists. The new formalisms of strange kinetics, on the other hand, seem designed for nonlinear processes taking place far from equilibrium. They are rich enough to accommodate the degrees of freedom needed for biological systems. Their patterns, which antedate the genetic code, are in principle applicable to the molecular events and the time sequences of biology. Even if these formalisms are only occasionally of service, their development may contribute to the reflectiveness of the participants and, thereby, rescue molecular biology from its blemishes.

\section{Philip R. Steinmetz}

Laboratory for Epithelial Transport and Kidney Physiology.

University of Connecticut School of Medicine,

Farmington, Connecticut 06030, USA

1. Shlesinger, M. F., Zaslavsky, G. M. \& Klafter, J. Nature 363, 31-37 (1993)

2. Maddox. J. Nature 363.13(1993)

3. Nicholis, G. \& Prigogine, I. Exporing Complexity 54 (W. H. Freeman and Co, New York, 1989)

4. Andersen, O. S. Silveira, J. E. N. \& Steinmetz, P. R. J. gen Physiol. 86. 215-234 (1985).

\section{Molecular biology}

SIR - I would like to comment on the article on the dark side of molecular biology ${ }^{1}$, in which ideas already put forward ${ }^{2}$ are developed further. It is true that molecular biology is a very powerful tool that gives rapid and simple answers the gene is or is not present, is or is not altered - to complex questions and that many researchers are satisifed with these answers.

The problem arises because quantitative measurements, contrary to what is possible in the field of physical chemistry, are concerned with open dynamic systems in which responses to different stimuli are linked and nonlinear. The study of such systems entails the use of powerful computational methods and either the determination of wealth of physicochemical parameters or recourse to simplification and approximations. This is often considered too complex and useless by many, not to say most, molecular biologists, partly because the striking results obtained, for example, in the field of human health may lead people to think that the reductionist approach used is not only sufficient but also the only one necessary and valid in all cases. This situation also obscures the fact that the state of a nonlinear system is not only a function of the initial condition (among which are enzyme or receptor quantity and characteristics) but also of its history: a given system may end up at two different steady states, depending on the nature of the stimuli it received.

As you say, quantification is long and difficult, too slow compared to the pace of the current descriptive race, and it also requires skills in the field of system dynamics. That is why the quantification effort comes mainly from chemical engineering laboratories, from people such as James Bailey $^{3}$ and Gregory Stephanopoulos ${ }^{4}$, with the help of molecular biologists.

Let us hope that more people will be convinced of the importance of quantification, the only way to a global vision and a complete understanding of living systems.

\section{Robert Lortle}

Biotechnology Research Institute,

National Research Council,

Montreal, Canada

1. Nature, 363, 13 (1993).

2. Nature, 355, 201 (1992)

3. Science, 252, 1668-1675 (1991)

4. Science, 252, 1675-1681 (2991)

\section{ESO's colony}

SIR - After reading of the negotiations between the European Southern Observatory (ESO) and the Chilean government about the use of the observatories (Nature 363, 384; 1993), as a Chilean scientist I could not avoid being struck by the similarities between the role of the ESO in Chile and the situation described by Joseph Conrad in Nostromo, his classic novel of colonialism in South America.

According to your report, the ESO in Chile appears to be the typical colonial enclave described by Conrad, acting independently of the local government, with policies that restrict the access of Chilean scientists to the laboratory and artificially lower the salaries of native workers and limit their professional advancement. This agency, moreover, expects the continuation of the advantageous concessions obtained from the Pinochet dictatorship. Nitrates, guano, silver and rubber may have been replaced by rights over land for laboratories and access to the sky, but the asymmetry of the relationship remains the same.

ESO's denial of guaranteed viewing time to Chilean astronomers, on the basis of their low numbers, is disingenuous and circular, as the only way to increase the number is to train more astronomers by, among other things, increasing their access to ESO. The fact that, after 30 years of ESO working in Chile, the Chilean astronomical community still remains small shows the unfairness of an arrangement that disregards the training of local scientists as a priority. It is difficult to imagine that any European country would tolerate the presence of a laboratory administered by Americans, to which the host country would be denied access.

Felipe C. Cabello

Department of Microbiology \& Immunology, New York Medical College,

Valhalla, New York 10595, USA 